\title{
Staphylococcal Endocarditis Presenting with a Renal Infarct in a Patient with Acute Lymphoblastic Leukemia
}

\author{
Meong Hi Son, M.D., Eun Sil Park, M.D., Ji-Hyun Seo, M.D., Jae-Young Lim, M.D., Chan-Hoo Park, \\ M.D., Hyang-Ok Woo, M.D. and Hee-Shang Youn, M.D.
}

Department of Pediatrics, Gyeongsang National University College of Medicine, Jinju, Korea

\begin{abstract}
We present here a patient with acute lymphoblastic leukemia (ALL) and who developed infective endocarditis during induction chemotherapy with prednisolone, L-asparaginase (Leunase ${ }^{\circledR}$ ), vincristine and adriamycin. The patient did not have a history of a central venous catheter. Sharp flank pain and fever occurred on the $25^{\text {th }}$ day of induction chemotherapy. In addition, a renal infarct and movable vegetations on the mitral valve were detected on the abdominal computed tomography (CT) and echocardiography. $S$. aureus was identified in the cultured blood. While the patient achieved remission, follow-up echocardiography revealed the vegetation had in-
\end{abstract}

\section{INTRODUCTION}

Cancer patients who are hospitalized and exhibit severe oral mucositis and catheter-related infections during an episode of profound neutropenia are at a higher risk for developing Staphylococcus aureus bacteremia (SBE) $(1,2)$. In addition, prior prophylaxis with quinolones can also be a risk factor for infection in these patients (2). Infective endocarditis is a life-threatening complication of SBE. The endocarditis from $S$. aureus infections, which is associated with mortality rates ranging from $25 \%$ to $40 \%$, primarily involves the left side of the heart and this is associated with thromboembolisms (TEs). The size (2) and primary location (3) of the endocarditis vegetations are important factors that are correlated with the development of these embolic complications. Notably, the treatment of cancer patients with L-asparaginase (L-ASP) is also associated with thromboembolic events due to the decreased hepatic production of multiple plasma proteins, including fibrinogen, plasminogen, alpha-2 antiplasmin, antithrombin, protein $\mathrm{C}$, protein $\mathrm{S}$ and some of the clotting factors $(4,5)$. Indeed, recent studies have shown an increased prevalence of thromboembolism (TE) in patients who have received L-ASP (6).

We report here on a rare complication of staphylococcal

Correspondence: Eun Sil Park, Department of Pediatrics, Gyeongsang National University College of Medicine, 90, Chilam-dong, Jinju 660-702, Korea. (Tel) 82-55-750-8829, (Fax) 82-55-752-9339, (E-mail) espark@gsnu.ac.kr

Received April 16, 2008, Accepted August 26, 2008 creased in size and an abscess pocket had developed despite the antibiotics and heparin therapy. Consequently, ten days after the diagnosis of infective endocarditis, a successful mitral valvuloplasty was performed without complications. The patient is currently on maintenance chemotherapy while in remission. (Cancer Res Treat. 2008;40:151-154)

Key Words: Precursor cell lymphoblastic leukemialymphoma, Staphylococcus aureus, Vegetation, Coagulation

endocarditis in a patient with acute lymphoblastic leukemia (ALL) and the patient had prior L-ASP treatment, but she had no history of a central venous catheter and she presented with consecutive infarcts and fever.

\section{CASE REPORT}

A 12-year-old girl who was diagnosed with pre-B cell type ALL was treated with intensive induction chemotherapy; this consisted of prednisolone $\left(60 \mathrm{mg} / \mathrm{m}^{2}\right)$, L-ASP $\left(6,000 \mathrm{IU} / \mathrm{m}^{2}\right.$ $3 /$ week), vincristine $\left(1.5 \mathrm{mg} / \mathrm{m}^{2}\right)$ and adriamycin $\left(25 \mathrm{mg} / \mathrm{m}^{2}\right)$. On the $25^{\text {th }}$ day after induction chemotherapy commenced, episodic fever developed along with epigastric discomfort. The abdomen was soft and flat without organomegaly, but sharp flank pain and fever developed three days later. Another physical examination revealed right costovertebral angle tenderness, but the abdomen remained soft without organomegaly. An abdominal CT scan was performed and a right renal infarct was noted (Fig. 1), so a physical examination was again performed. The cardiac examination revealed a grade III/VI holosystolic murmur at the apex of the heart. The vital signs were as follows: blood pressure $100 \sim 110 / 60 \sim 70 \mathrm{mmHg}$, heart rate $84 \sim$ $108 / \mathrm{min}$, respiration rate $14 \sim 20 / \mathrm{min}$ and body temperature 36 $\sim 38^{\circ} \mathrm{C}$. Rales were not heard upon auscultation of the lungs and no evidence of splinter hemorrhages or petechiae on the extremities was observed. In addition, there were no signs of Osler's nodes or Janeway's lesions. The laboratory findings were as follows: hemoglobin $9.4 \mathrm{~g} / \mathrm{dl}$, hematocrit $27 \%$, WBC $5,000 /$ ul (seg 89.6\%) and platelets 55,000/ul. The fibrinogen levels were $185 \mathrm{mg} / \mathrm{dl}$, the anti-thrombin III levels were $64 \%$ $(80 \sim 120)$, the protein $\mathrm{C}$ levels were $62 \% \quad(72 \sim 160)$, the 

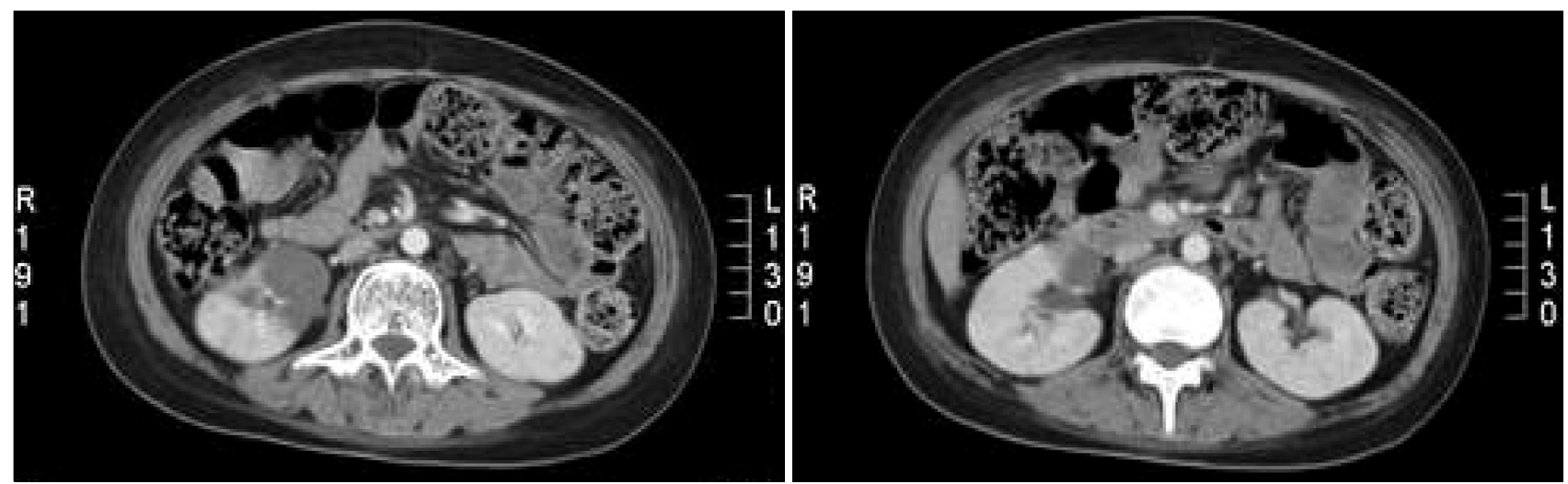

Fig. 1. An abdominal CT showed a right renal infarct on the 1st day.
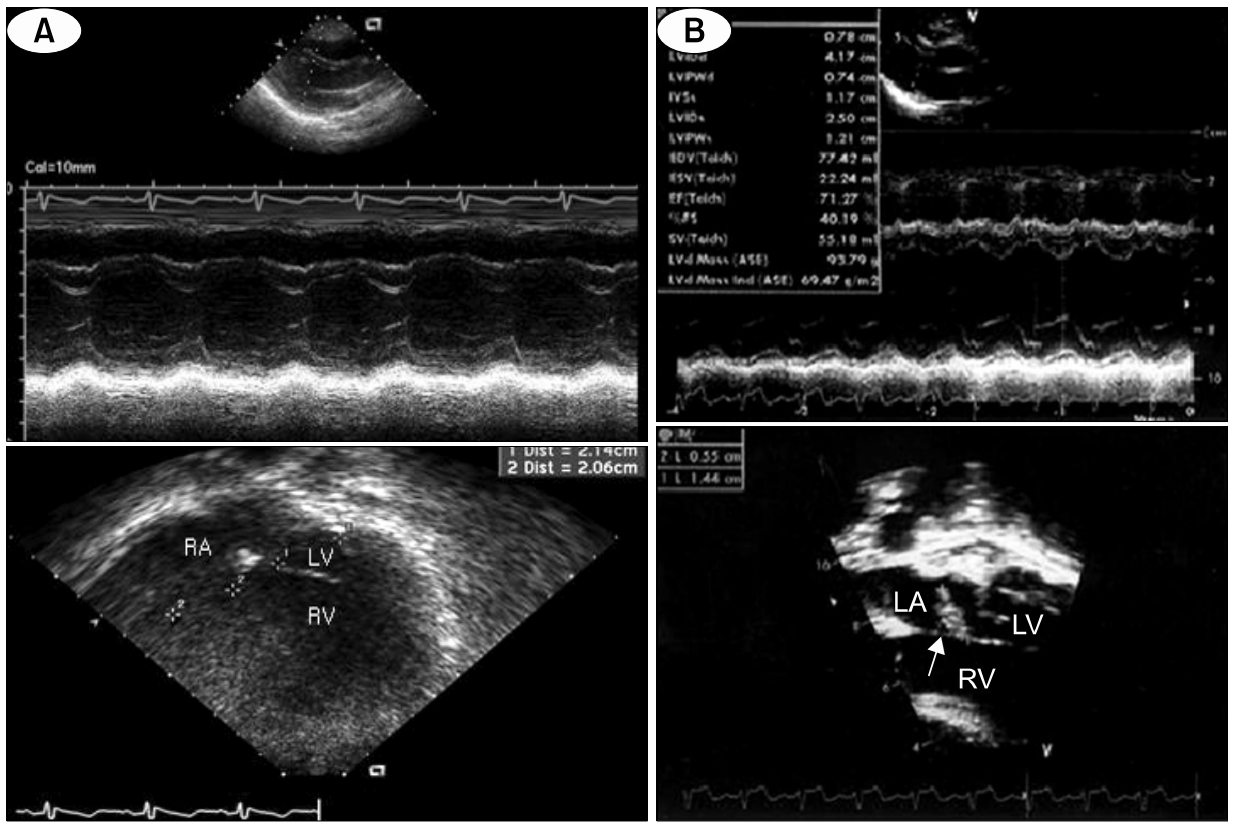

Fig. 2. TTE findings. (A) A baseline TTE that was performed before chemotherapy commenced. (B) The TTE revealed infective endocarditis. The size of the vegetation was $14 \times 5.5$ $\mathrm{mm}$.

protein S levels were $51 \%(60 \sim 150)$, the erythrocyte sedimentation rate (ESR) was $2 \mathrm{~mm} / \mathrm{hr}$ and the C-reactive protein (CRP) level was $101.1 \mathrm{mg} / \mathrm{dl}$.

These observations suggested that the patient had a venous thrombosis and so we administered low molecular weight heparin. Transthoracic echocardiography (TTE) revealed a vegetationlesion in the anterior leaflet of the mitral valve that was $12 \times 5.5$ $\mathrm{mm}$ in diameter and this was accompanied by mild mitral regurgitation. The baseline TTE performed before chemotherapy had not revealed any abnormalities (Fig. 2A). In addition, $S$. aureus was isolated after culturing the peripheral blood for nine days. Antibiotic sensitivity testing revealed that the organisms were sensitive to oxacillin and vancomycin, but not to penicillin-G. The patient had no history of a central catheter. These observations were consistent with the diagnosis of both infective endocarditis and a thromboembolism. Treatment with antibiotics (vancomycin and gentamicin) and heparin was then done. The patient's fever subsided within three days. However, six days after antibiotic treatment was initiated, the patient complained of abdominal and toe pain. Multiple splenic infarcts were newly detected upon the 3-dimensional abdominal CT scan (Fig. 3). Upon follow-up, the TTE revealed that the vegetation-lesion on the valve leaflet had increased in size (it was now $14 \times 5.5 \mathrm{~mm}$ in diameter) and a perivalvular abscess pocket was newly detected (Fig. 2B). Since medical treatment had failed, urgent surgical intervention was planned. During surgery, debridement and curettage of the abscess pocket was performed. In addition, the mitral valve was reconstructed with a Carpentier-Edward mitral valve prosthesis. Postoperatively, the patient was treated for six weeks with the antibiotic vancomycin. The patient recovered without any complications. Six weeks later, systemic chemotherapy (consolidation) was initiated. 

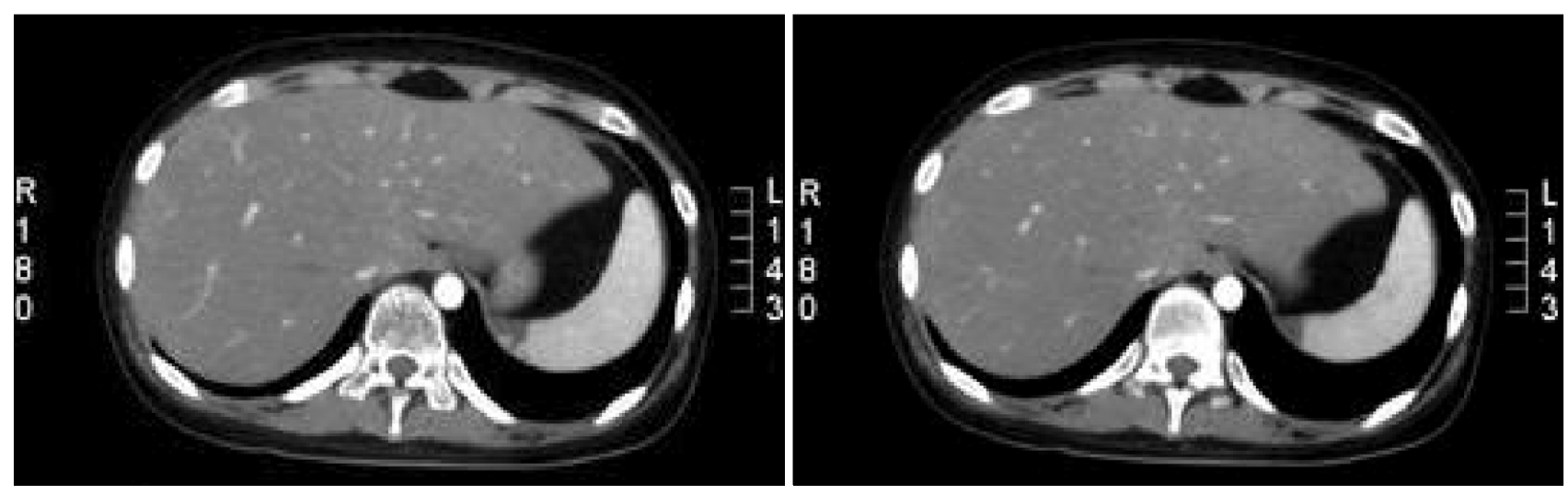

Fig. 3. Eight days after the first CT, a second abdominal CT revealed multiple splenic infarcts.

\section{DISCUSSION}

We did not initially realize that infective endocarditis was the cause of the fever in our patient as endocarditis does not commonly occur in patients with leukemia, even though these patients are prone to develop fever and bacteremia. Therefore, when our patient first presented with fever and abdominal pain, we did not evaluate the heart sounds, rather, we focused on the abdominal CT. It was only after the TE was detected in the right kidney that we evaluated the heart. Our patient had two episodes of TE, with the second episode occurring while the patient was receiving medical treatment for the first episode. That the patient had leukemia meant that she had an elevated risk of developing TEs. This risk was probably further increased by her prior treatment with L-ASP, as L-ASP treatment is well known to increase the risk that leukemia patients will develop a TE $(4,5,7)$. Moreover, the presence of the SBE may have even further increased the chance that the patient developed a TE $(8 \sim 12)$. This is because sepsis and septic shock with $S$. aureus infections can lead to disseminated intravascular coagulation and multiorgan dysfunction syndrome. The cell wall components of $S$. aureus, namely, peptidoglycan and lipoteichoic acid, have been shown to induce human monocytes to express tissue factor, which initiates the coagulation cascade by activating factor VII $(8,9)$. Factor VII is a potent procoagulant and it activates factors IX and X. These factors together lead to the activation of thrombin. Previous studies have shown that the tissue factor expressed by monocytes is a major stimulus for the formation of vegetations in patients with bacterial endocarditis $(10,11)$. In addition, $S$. aureus spp. activates the expression of a variety of endothelial (E-selectin), intercellular (ICAM-1) and vascular (VCAM-1) adhesion molecules. These adhesion molecules are likely to play important roles in both the pathogenesis of infective endocarditis and the occurrence of thromboembolic events (12).

L-ASP is widely used for the treatment of acute lymphoblastic leukemia and it is well known for its prothrombotic effects and its association with TE events. These events are thought to be due to the decreased hepatic production of multiple plasma proteins, including fibrinogen, plasminogen, alpha-2 antiplasmin, antithrombin, protein $\mathrm{C}$, protein $\mathrm{S}$ and some of the clotting factors $(13,14)$. Previous studies have shown that the prevalence of TEs in children with ALL and who receive L-ASP ranged from $1 \%$ to $14 \%$. However, the PARKAA (Prophylactic Antithrombin Replacement in Kids with ALL Treated with Asparaginase) study showed that the prevalence of TE in children with ALL and who receive L-ASP was as high as $36.7 \%$ (6). This increase in prevalence is probably because the asymptomatic patients were evaluated radiologically, along with the symptomatic patients.

The two major clinical criteria for the diagnosis of SBE are proven bacteremia or fungemia within a given time interval, in addition to echocardiographic evidence (15). The findings with regard to our patient satisfied these two criteria. Endocarditis due to $S$. aureus in non-addicts primarily involves the left side of the heart and this is associated with high mortality rates that range from $25 \%$ to $40 \%$. Early surgical intervention must be considered to avoid TEs and mortality (15). The indications for surgical intervention include large vegetations $(>10 \mathrm{~mm}$ in diameter, especially on the anterior mitral leaflet), embolic events during the first two weeks of antimicrobial therapy, an increase in vegetation size despite appropriate antimicrobial therapy, severe valvular insufficiency, abscess cavities or pseudoaneurysms, valvular perforation or dehiscence, and evidence of decompensated heart failure (15).

In our case, the antibiotics tazobactam and amikacin were administered IV after fever developed, and vancomycin was added after the patient's endocarditis was diagnosed. Treatment with low molecular weight heparin was also initiated after the renal infarct was identified on CT. However, even though our patient received the appropriate medical management, two embolic episodes developed, namely, a renal infarct and a splenic infarct, within two weeks of the initial diagnosis and treatment. In addition, clinical deterioration was noted on the follow up echocardiography, as the formation of an abscess was detected. Therefore, surgical intervention was considered necessary to improve the outcome. After mitral valvular reconstruction, six weeks of vancomycin was administered to treat the complicated infective endocarditis.

Thromboembolic complications due to infective endocarditis are rare events in patients with leukemia, even though the risk 
for infection is high in neutropenic patients during chemotherapy. This patient had several risk factors that increased the chance of developing these complications, including the fact that she had leukemia and she received L-ASP chemotherapy. A higher prevalence of TEs in patients who receive L-ASP therapy with a central venous catheter has been reported (6). However, this is the first report of a TE occurring in a patient who received L-ASP therapy and she developed SBE, but she lacked a history of a central catheter.

This case illustrates the importance of considering SBE when diagnosing leukemia patients who present with a fever and no obvious focus of infection. In addition, prior treatment of bacteremic patients with L-ASP should alert clinicians to the possibility that these patients may have a higher risk of developing thromboembolic events, and even if there is no history of a central venous catheter. Further, if the patient does not respond to medical treatment, then surgery should be considered to improve the outcome of the patient.

\section{REFERENCES}

1. Gonzalez-Barca E, Carratala J, Mykietiuk A, Fernandez-Sevilla A, Gudiol F. Predisposing factors and outcome of Staphylococcus aureus bacteremia in neutropenic patients with cancer. Eur J Clin Microbiol Infect Dis. 2001;20:117-9.

2. Mugge A, Daniel WG, Frank G, Lichtlen PR. Echocardiography in infective endocarditis: reassessment of prognostic implications of vegetation size determined by the transthoracic and the transesophageal approach. J Am Coll Cardiol. 1989; 14:631-8.

3. Rohmann S, Seifert T, Erbel R, Jakob H, Mohr-Kahaly S, Makowski $\mathrm{T}$, et al. Identification of abscess formation in native-valve infective endocarditis using transesophageal echocardiography: implications for surgical treatment. Thorac Cardiovasc Surg. 1991;39:273-80.

4. Alberts SR, Bretscher M, Wiltsie JC, O'Neill BP, Mokri B, Witzig TE. Thrombosis related to the use of L-asparaginase in adults with acute lymphoblastic leukemia: a need to consider coagulation monitoring and clotting factor replacement. Leuk Lymphoma 1999;32:489-96.

5. Shapiro AD, Clarke SL, Christian JM, Odom LF, Hathaway WE. Thrombosis in children receiving L-asparaginase. Determining patients at risk. Am J Pediatr Hematol Oncol. 1993;15: 400-5.

6. Mitchell LG, Andrew M, Hanna K, Abshire T, Halton J,
Anderson $\mathrm{R}$, et al. A prospective cohort study determining the prevalence of Thrombotic Events in Children with Acute Lymphoblastic Leukemia and a Central Venous Line Who are Treated with L-Asparaginase: results of the Prophylactic Antithrombin Replacement in Kids with Acute Lylmhoblastic Leukemia Treated with Asparaginase (PARKAA) Study. Cancer. 2003;97:508-16.

7. Unal S, Varan A, Yalcin B, Buyukpamukcu M, Gurgey A. Evaluation of thrombotic children with malignancy. Ann Hematol. 2005;84:395-9.

8. Mattsson E, Hartung T, Morath S, Egesten A. Highly purified lipoteichoic acid from Staphylococcus aureus induces procoagulant activity and tissue factor expression in human monocytes but is a weak inducer in whole blood: comparison with peptidoglycan. Infect Immun. 2004;72:4322-6.

9. Mattsson E, Herwald H, Bjorck L, Egesten A. Peptidoglycan from staphylococcus aureus induces tissue factor expression and procoagulant activity in human monocytes. Infect Immun. 2002;70:3033-9.

10. Drake TA, Rodgers GM, Sande MA. Tissue factor is a major stimulus for vegetation formation in enterococcal endocarditis in rabbits. J Clin Invest. 1984;73:1750-3.

11. Veltrop MH, Beekhuizen H. Monocytes maintain tissue factor activity after cytolysis of bacteria-infected endothelial cells in an in vitro model of bacterial endocarditis. J Infect Dis. 2002;186:1145-54.

12. Korkmaz S, Ileri M, Hisar I, Yetkin E, Kosar F. Increased levels of soluble adhesion molecules, E-selectin and P-selectin, in patients with infective endocarditis and embolic events. Eur Heart J. 2001;22:874-8.

13. Alberts SR, Bretscher M, Wiltsie JC, O'Neill BP, Mokri B, Witzig TE. Thrombosis related to the use of $\mathrm{L}$-asparaginase in adults with acute lymphoblastic leukemia: a need to consider coagulation monitoring and clotting factor replacement. Leuk Lymphoma. 1999;32:489-96.

14. Shapiro AD, Clarke SL, Christian JM, Odom LF, Hathaway WE. Thrombosis in children receiving L-asparaginase. Determining patients at risk. Am J Pediatr Hematol Oncol. 1993;15: 400-5.

15. Baddour LM, Wilson WR, Bayer AS, Fowler VG Jr, Bolger $\mathrm{AF}$, Levison ME, et al. Infective endocarditis: diagnosis, antimicrobial therapy, and management of complications: a statement for healthcare professionals from the Committee on Rheumatic Fever, Endocarditis, and Kawasaki Disease, Council on Cardiovascular Disease in the Young, and the Councils on Clinical Cardiology, Stroke, and Cardiovascular Surgery and Anesthesia, American Heart Association: endorsed by the Infectious Diseases Society of America. Circulation. 2005;111: e394-434. 